\title{
Ultrasound Findings were Associated With Radiographic Changes, But Not Clinical and Functional Outcomes in Hand Osteoarthritis
}

\author{
Seong-Kyu Kim, M.D., Ph.D., Ui Hong Jung, M.D., Ji-Won Kim, M.D., Ph.D., Jung-Yoon Choe, M.D., Ph.D. \\ Division of Rheumatology, Department of Internal Medicine, Daegu Catholic University School of Medicine, Daegu, Korea
}

Objective. There is a debate over the relevance of ultrasound abnormalities to the pain, functional impairment, and radiologic severity in hand osteoarthritis (OA). This study aims to determine the association between ultrasound abnormalities and clinical, functional, and radiographic measures in hand OA. Methods. A total of 66 patients was consecutively enrolled. All patients with gray-scale synovitis, joint effusion, and osteophytes were examined by ultrasound for 20 hand joints. Radiographic changes in both hands were evaluated by the Kellgren-Lawrence (K-L) grading system and were described as total radiographic severity score and number of affected joints. Other measures were also assessed, including each patient's visual analogue scale for pain, the Functional Index for Hand Osteoarthritis for functional disability, and grip and pinch strength for hand muscle strength. Results. In total, 10 patients with gray-scale synovitis, 35 with joint effusion, and 66 with osteophytes were detected in hand OA scans on ultrasound. Osteophytes on ultrasound were significantly associated with total radiographic severity score and number of affected joint $(r=0.293, p=0.003$ and $r=0.336, p<0.001$, respectively). In addition, there were weak associations of synovitis and joint effusion with radiographic changes. Patients with higher total radiographic severity score showed larger number of ultrasound-detected abnormalities, such as synovitis, joint effusion, and osteophytes $(p=0.011, p=0.002$, and $\mathrm{p}<0.001$, respectively). Conclusion. This study shows that ultrasound findings, especially osteophytes, were associated with radiographic changes based on K-L grade, but not clinical and functional status in hand OA. (J Rheum Dis 2021;28:17-24)

Key Words. Osteoarthritis, Hand, Radiography, Ultrasound, Kellgren-Lawrence grade

\section{INTRODUCTION}

Hand osteoarthritis (OA) is a degenerative articular disease that ultimately results in pain, deformity, functional disability, and poor quality of life [1,2]. It is a heterogeneous disease characterized by a multifactorial etiology that presents with distinct clinical symptoms and radiographic findings. Hand OA is mainly defined based on clinical and/or radiographic features [3]. Epidemiologic studies of the general population have shown that the prevalence and incidence of hand $\mathrm{OA}$ and its clinical and radiographic severity tend to increase with age $[4,5]$.

Standardized outcome instruments to assess complex clinical, functional, and radiographic features in hand OA have been developed for several decades. The Outcome Measures in Rheumatology and the Osteoarthritis Research Society International (OARSI) Task Force on Clinical Trials Guidelines suggested three core set clinical measures, pain, physical function, and patient global assessment, for use in clinical trials [6,7]. In addition, diverse musculoskeletal imaging modalities for evaluating hand joints, such as conventional radiography, ultrasound, and magnetic resonance imaging (MRI), are available in clinical practice [8]. Specifically, ultrasound has been well recognized to be a valid and reliable tool to detect synovial inflammation, osteophytes, and joint effu-

\footnotetext{
Received : July 29, 2020, Revised : (1st) August 28, 2020, (2nd) September 18, 2020, Accepted : September 18, 2020

Corresponding to : Seong-Kyu Kim (10) http://orcid.org/0000-0002-7780-0167

Division of Rheumatology, Department of Internal Medicine, Daegu Catholic University School of Medicine, 33 Duryugongwon-ro 17-gil, Nam-gu, Daegu 42472, Korea. E-mail : kimsk714@cu.ac.kr
}

Copyright (C) 2021 by The Korean College of Rheumatology.

This is an Open Access article, which permits unrestricted non-commerical use, distribution, and reproduction in any medium, provided the original work is properly cited. 
sion in the small joints of the hands [8,9]. Some studies evaluated the association of ultrasound abnormalities with clinical features, functional status, and radiographic findings in hand OA [10-14]. Their relevance is still debated according to study population and outcome measures.

There is a lack of data on whether ultrasound findings have a close relationship with diverse outcome measures of hand OA in clinical practice. Therefore, we evaluated the association between ultrasound findings and pain, physical function, and radiologic changes in symptomatic hand OA.

\section{MATERIALS AND METHODS}

\section{Study population}

All patients enrolled in this study fulfilled the American College of Rheumatology classification criteria for hand OA [3]. A total of 66 patients with hand OA was enrolled from an outpatient clinic in Daegu Catholic University Medical Center. Subjects diagnosed as having other inflammatory or autoimmune rheumatic diseases including rheumatoid arthritis, systemic lupus erythematosus, crystal-induced arthritis, and psoriatic arthritis were excluded from this study. This study was approved by the Institutional Review Board (CR-20-139-L).

\section{Assessment of clinical information}

Demographic data on age, sex, height, weight, body mass index (BMI, $\mathrm{kg} / \mathrm{m}^{2}$ ), symptom duration (months), systolic blood pressure (SBP, $\mathrm{mmHg}$ ), and diastolic blood pressure (DBP, $\mathrm{mmHg}$ ) were identified. At the visit, acute phase reactants such as erythrocyte sedimentation rate (ESR, $\mathrm{mm} / \mathrm{hr}$ ) and C-reactive protein (CRP, $\mathrm{mg} / \mathrm{L}$ ) were evaluated. For analysis of function and pain for hand OA, we evaluated physical function using the Functional Index for Hand Osteoarthritis (FIHOA), which results in scores from 0 to 30 [15]. A Korean version of FIHOA, which was validated in Korean patients with hand OA, was used in this study [16]. In addition, pain status was assessed by the 100-mm Visual Analogue Scale (VAS) [17].

\section{Assessment of radiographic findings}

Radiographic changes in both hands were assessed according to the Kellgren-Lawrence (K-L) grading system [18]. We assessed a total of 20 joints in both hands, including the 2 nd to 5 th distal interphalangeal (DIP) joints, the 2nd to 5th proximal interphalangeal (PIP) joints, the thumb interphalangeal (IP) joint, and the first carpometacarpal (CMC) joint. For the overall radiographic severity of each patient, the most severe grade among total 20 joints was described as K-L grade, ranged from grade I to grade IV. Total radiographic severity score was defined as the sum of the K-L grading scores of the 20 individual hand joints, resulting in scores ranging from 0 to 80 $[5,19]$. The number of affected joints on conventional radiography is the number of the 20 joints with K-L grade of 1 or higher, leading to a score from 0 to 20 . All radiographic digital images of hand joints were evaluated by one rheumatologist (UH Jung).

\section{Assessment of ultrasound findings}

Ultrasound findings evaluated in the hand OA patients were gray-scale synovitis, joint effusion, and osteophytes [9], and the ultrasound examinations were performed using an ACUSON S2000 Ultrasound System (Siemens Healthineers, Seoul, Korea) with a 5 to $14 \mathrm{MHz}$ linear transducer (14L5) by one rheumatologist (UH Jung). Ultrasound images were obtained by examining the sagittal and lateral aspects of the 2nd to 5th DIP joints, the 2nd to 5th PIP joints, the thumb IP joint, and the first CMC joint [9]. The ultrasound-detected abnormalities such as gray-scale synovitis, joint effusion, and osteophytes were assessed if present in individual joints of both hands without regard to severity. In addition, the number of joints with each abnormality was calculated and scored from 0 to 20 . The ultrasound definitions for effusion, gray-scale synovitis, and osteophytes used in our previous study were applied [20]. Intraclass correlation coefficient with $95 \%$ confidential interval (CI) for intra-observer reliability was calculated; $0.950(95 \% \mathrm{CI}$ $0.888 \sim 0.978$ ) for K-L grade, 0.994 (95\% CI 0.987 0.997) for total radiographic severity score, 0.984 (95\% CI $0.964 \sim 0.993)$ for number of affected joint, 0.957 (95\% CI 0.904 0.981) for synovitis, 0.871 (95\% CI $0.724 \sim 0.942)$ for joint effusion, and 0.798 (95\% CI $0.588 \sim 0.907$ ) for osteophyte.

\section{Assessment of muscle strength in both hands}

Muscle strength in both hands was assessed using grip strength evaluated by dynamometer (Jamar Hydraulic Hand Dynamometer, Nottinghamshire, UK) and pinch strength for the 1st and 2nd fingers measured by a pinch gauge (B \& L Engineering, Tustin, CA, USA). After measuring the pinch and grip strengths for both hands, they 
were expressed as mean values for each measure.

\section{Statistical analysis}

Data were reported as median (interquartile range [IQR]) for continuous variables or number (percentage [\%]) for categorical variables. All statistical tests were performed by nonparametric analysis after testing for normality of the distribution using the KolmogorovSmirnov test. Correlation of ultrasound abnormalities with clinical, laboratory, and radiographic findings was calculated by Spearman's correlation method. These correlation analyses were confirmed after adjustments for confounding factors of sex, age, and symptom duration. Comparisons of the numbers of ultrasound abnormalities such as synovitis, joint effusion, and osteophytes according to total radiographic severity score ( $\leq 10$ vs. $>10)$ were measured by Mann-Whitney U-tests. The level of statistical significance was less than 0.05. Analyses were performed using IBM SPSS Statistics 19.0 (IBM Corp., Armonk, NY, USA).

\section{RESULTS}

\section{Baseline demographic and clinical characteristics}

A total of 66 patients with hand OA was enrolled in this

Table 1. Baseline characteristics of demographic, clinical, and laboratory findings in enrolled subjects

\begin{tabular}{lc}
\hline \multicolumn{1}{c}{ Variable } & Value \\
\hline Age $(\mathrm{yr})$ & $57.5(52.0 \sim 63.3)$ \\
Sex, female & $61(92.4)$ \\
BMI $\left(\mathrm{kg} / \mathrm{m}^{2}\right)$ & $23.4(21.1 \sim 25.4)$ \\
SBP $(\mathrm{mmHg})$ & $125.0(114.0 \sim 135.0)$ \\
DBP $(\mathrm{mmHg})$ & $73.0(67.0 \sim 79.3)$ \\
Past history & $14(21.2)$ \\
Hypertension & $6(9.1)$ \\
Diabetes mellitus & $2.0(0.2 \sim 12.0)$ \\
Symptom duration $(\mathrm{mo})$ & $140(8.0 \sim 24.3)$ \\
ESR $(\mathrm{mm} / \mathrm{hr})$ & $0.60(0.06 \sim 0.80)$ \\
CRP $(\mathrm{mg} / \mathrm{L})$ & $19.6(14.9 \sim 24.6)$ \\
Grip strength & $5.5(4.3 \sim 6.5)$ \\
Pinch strength & $5.0(2.0 \sim 8.0)$ \\
FIHOA & $35.0(22.0 \sim 50.5)$ \\
Patient VAS &
\end{tabular}

Data were described as median (interquartile range) or number (\%). BMI: body mass index, SBP: systolic blood pressure, DBP: diastolic blood pressure, ESR: erythrocyte sedimentation rate, CRP: C-reactive protein, FIHOA: Functional Index for Hand Osteoarthritis, VAS: visual analogue scale. study, and 61 of them were female (91\%). The median age of the patients was 57.5 years (IQR: 52.0 63.3 years). Other demographic variables such as BMI, SBP, DBP, and past medical history are shown in Table 1, combined with laboratory parameters such as ESR and CRP as well as symptom duration. Results for hand muscle strength, physical function, and pain using grip and pinch strength, FIHOA, and VAS are also described.

\section{Radiographic and ultrasound data}

The median value of total radiographic severity score at 20 joints of both hands was 5.0 (IQR: 2.0 14.0) (Table 2). Among 20 hand joints, any kind of radiographic abnormality presented on the K-L grading system was found in approximately four joints per patient (IQR: $2.0 \sim 8.0$ ). Only 10 out of 66 patients showed gray-scale synovitis on ultrasound, involving 1 to 3 joints per patient. Joint effusion was detected in 35 patients (53.0\%) with a median of 1.0 joint (IQR: $0.0 \sim 2.0$ ), presenting with 1 to 8 joints per patient. Osteophytes were detected in all patients on ultrasound. Ultrasound-detected osteophytes were found in 2 to 18 joints in individual patients, with a median of 10 joints (IQR: 7.0 15.3).

\section{Correlation between ultrasound findings and clinical, laboratory, and radiographic variables}

In the Spearman's correlation analysis of the association of ultrasound findings with clinical and laboratory parameters, osteophytes were significantly associated with age, symptom duration, and grip strength $(r=0.301, p=0.014$;

Table 2. Baseline characteristics of radiologic and ultrasound findings in enrolled subjects

\begin{tabular}{lc}
\hline \multicolumn{1}{c}{ Variable } & Value \\
\hline Radiographic findings & \\
K-L grade & \\
Grade I & $27(40.9)$ \\
Grade II & $19(28.8)$ \\
Grade III & $11(16.7)$ \\
Grade IV & $9(13.6)$ \\
Total radiographic severity score $(0 \sim 80)$ & $5.0(2.0 \sim 14.0)$ \\
Number of affected joints & $4.0(2.0 \sim 8.0)$ \\
Ultrasound findings & \\
Synovitis & $10(15.2)$ \\
Effusion & $35(53.0)$ \\
Osteophyte & $66(100.0)$ \\
\hline
\end{tabular}

Data were described as number (\%) or median (interquartile range). K-L grade: Kellgren-Lawrence grade. 
$\mathrm{r}=0.302, \mathrm{p}=0.014$; and $\mathrm{r}=-0.290, \mathrm{p}=0.018$, respectively), as shown in Table 3. In the analysis of the association of ultrasound abnormalities with radiographic findings, numbers of joints with gray-scale synovitis, joint effusion, and osteophytes were statistically associated with total radiographic severity score $(r=0.260, p=0.035$; $\mathrm{r}=0.453, \mathrm{p}<0.001$; and $\mathrm{r}=0.601, \mathrm{p}<0.001$, respectively).
In addition, joint effusion and osteophytes on ultrasound were related to the number of affected joints $(p<0.001$ and $\mathrm{p}<0.001$, respectively), but synovitis was not $(\mathrm{p}=$ $0.106)$.

After adjustment for sex, age, and symptom duration, all ultrasound findings were associated with total radiographic severity score and number of affected joints

Table 3. Correlation between ultrasound findings and clinical, laboratory, and radiographic variables

\begin{tabular}{lcll}
\hline \hline \multirow{2}{*}{ Variable } & & Ultrasound findings & \\
\cline { 2 - 4 } & No. of synovitis & No. of joint effusion & No. of osteophytes \\
\hline Age & $r=-0.119, p=0.343$ & $r=0.040, p=0.748$ & $r=0.301, p=0.014$ \\
BMI & $r=-0.229, p=0.114$ & $r=0.015, p=0.920$ & $r=0.098, p=0.502$ \\
Symptom duration & $r=-0.030, p=0.810$ & $r=0.086, p=0.490$ & $r=0.302, p=0.014$ \\
ESR & $r=-0.047, p=0.706$ & $r=-0.106, p=0.397$ & $r=0.008, p=0.951$ \\
CRP & $r=-0.165, p=0.184$ & $r=-0.117, p=0.351$ & $r=0.080, p=0.522$ \\
FIHOA & $r=0.065, p=0.604$ & $r=0.078, p=0.534$ & $r=0.048, p=0.704$ \\
Grip strength & $r=-0.163, p=0.604$ & $r=-0.241, p=0.051$ & $r=-0.290, p=0.018$ \\
Pinch strength & $r=-0.030, p=0.191$ & $r=-0.127, p=0.311$ & $r=-0.189, p=0.130$ \\
Patient VAS & $r=0.102, p=0.415$ & $r=0.045, p=0.719$ & $r=0.122, p=0.328$ \\
Total radiographic score & $r=0.260, p=0.035$ & $r=0.453, p<0.001$ & $r=0.601, p<0.001$ \\
Number of affected joints & $r=0.201, p=0.106$ & $r=0.462, p<0.001$ & $r=0.592, p<0.001$ \\
\hline
\end{tabular}

Data were described as correlation coefficient (r). BMI: body mass index, ESR: erythrocyte sedimentation rate, CRP: C-reactive protein, FIHOA: Functional Index for Hand Osteoarthritis, VAS: visual analogue scale.
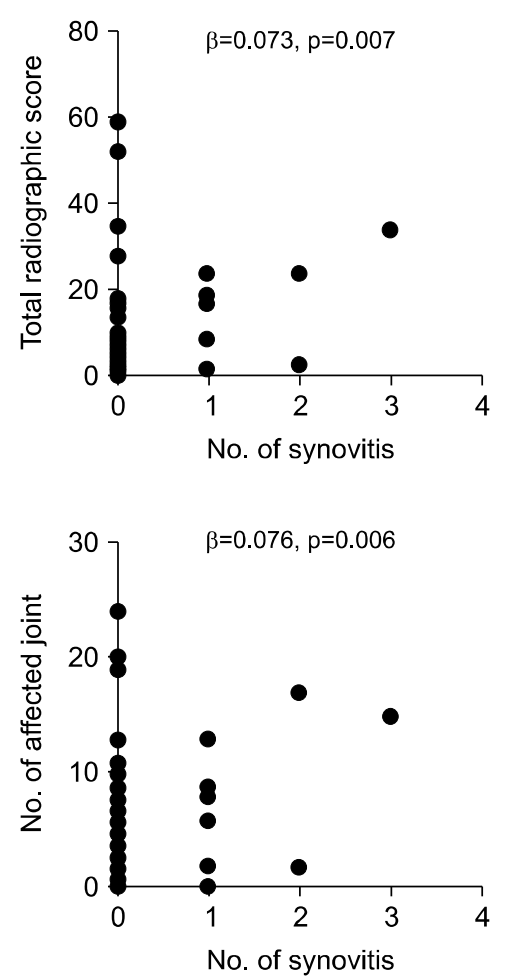
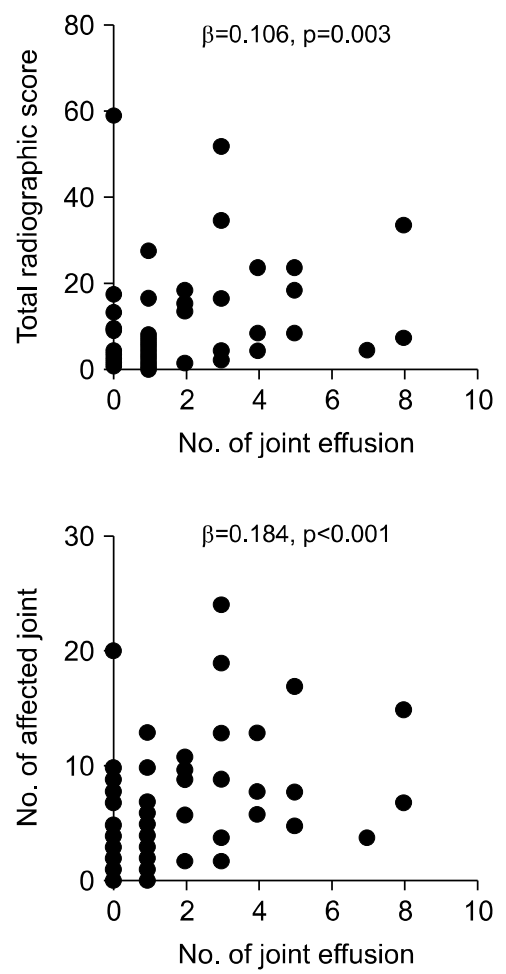
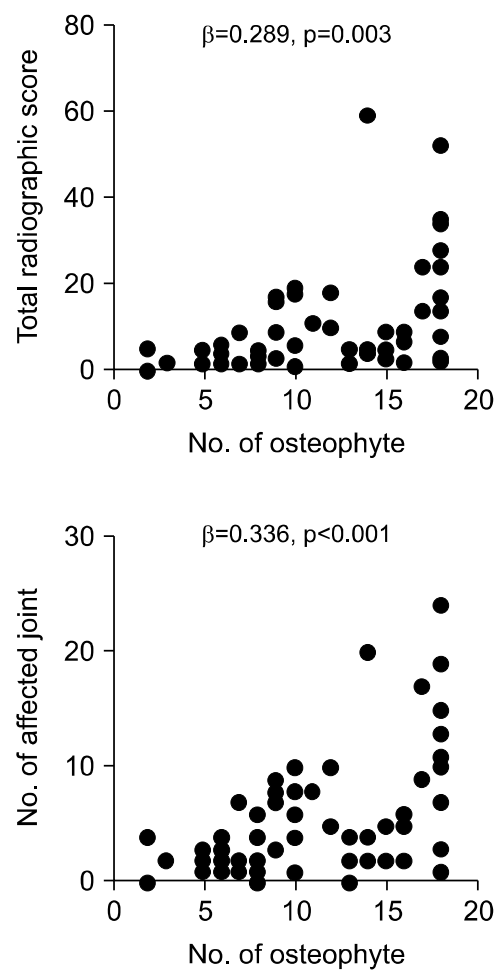

Figure 1. Correlation between radiographic changes and ultrasound abnormalities. p-values were calculated after adjustment with sex, age, and symptom duration. $\beta$ : regression coefficient in multivariate regression analysis. 
(Figure 1). However, numbers of joints with synovitis and joint effusion on ultrasound had only weak association with radiographic changes, although it was statistically significant. In addition, there was no association between ultrasound findings and grip strength (data not shown). Number of joints with synovitis, joint effusion, and osteophytes on ultrasound in patients $(n=18)$ with higher total radiographic severity scores $(>10)$ was markedly larger than those in patients $(n=48)$ with lower total radiographic severity scores $(\leq 10)(\mathrm{p}=0.011, \mathrm{p}=0.002$, and $\mathrm{p}<0.001$, respectively; Figure 2 ).

\section{DISCUSSION}

Ultrasound is an important and useful imaging modality for diagnosis and monitoring of treatment response in hand OA $[8,9]$. Recently, the relationship between clinical symptoms/signs and ultrasound-based findings has been investigated in several studies, but they showed inconsistent results [10-14]. Here, our study assessed the association of clinical, functional, and radiographic findings with ultrasound-detected abnormalities, such as gray-scale synovitis, joint effusion, and osteophytes, in hand OA. We found that osteophytes on ultrasound were significantly associated with radiographic changes but not with other clinical, laboratory, or functional measures. Earlier studies have investigated the relationship between ultrasound abnormalities and radiographic findings. Osteophyte scores on ultrasound were associated with K-L scores, OASRIS osteophyte scores, OARSI joint space narrowing, and Eaton subluxation in hand OA $[10,14]$. In contrast, synovitis and power Doppler signal had no relation with any radiographic grading systems in hand $\mathrm{OA}$ [10]. Similarly, Roux et al. [21] found no association of MRI-based synovitis with any radiographic scores includ- ing Verbruggen grade, K-L grade, and Kallman scores in patients with painful hand OA. This suggests discordance between ultrasound-detected synovitis and osteophytes evident upon conventional radiography. However, our study observed a weak association between synovitis and joint effusion and radiographic changes. Considering the reason for an association between gray-scale synovitis and radiographic changes, it has been established that mesenchymal stem cells and macrophage-like cells in the synovial membrane are the main cellular components in the process of osteophyte formation in OA [22]. It could be explained that synovial inflammation in mild or early synovitis is partly responsible for progression to osteophyte formation in hand OA. The inconsistency of the study results may also be due to the study design, that is, the number of joints evaluated, the ultrasound assessment, and the radiography scoring. In addition, other confounding components such as age and mechanical stimuli also contribute to osteophyte formation and were considered as independent factors. Further research is needed to investigate the relevance between ultrasound and conventional radiography findings.

Conventional radiography is a standard technique to assess morphologic abnormalities such as osteophytes, joint space narrowing, subchondral sclerosis, and bone erosion in hand OA [23]. However, it has been recognized that radiographic severity in hand OA did not parallel its symptoms or signs in large study populations $[24,25]$. On the other hand, patients with symptomatic joints had more frequent ultrasound abnormalities, such as gray-scale synovitis, osteophytes, and power Doppler signals, than control subjects without joint pain [12]. In addition, ultrasound provides more detailed information about changes of inflammatory joints than do clinical examinations for synovitis [13]. This might suggest that the
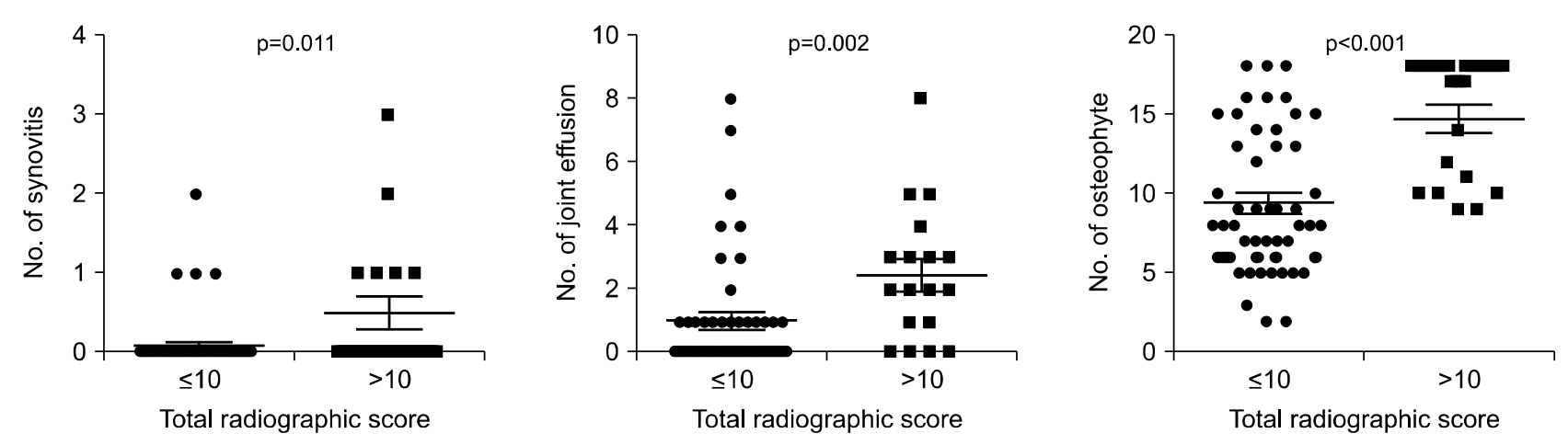

Figure 2. Comparison of scores for each ultrasound abnormality according to Kellgren-Lawrence grade. 
abnormalities observed by ultrasound assessment are much more related to the symptoms of hand OA than those found via radiography. Kortekaas et al. [11] demonstrated that gray-scale synovitis, effusion, and synovial thickening were positively associated with pain upon palpation. In a study of thumb-base OA, power Doppler activity detected by ultrasound had a marked correlation with pain VAS score (adjusted $\beta=11.29, \mathrm{p}=0.022$ ) but not with synovitis, osteophytes, or bone erosion [10]. However, other clinical measures including pinch strength, grip strength, and FIHOA were not related to ultrasound pathologies. In another perspective, an ultrasound study of 36 patients with hand OA revealed that the degree of symptoms in the affected joints was not associated with any ultrasound-detected pathology including gray-scale synovitis, synovitis with power Doppler activity, and osteophytes [12]. Consistently, our study also found no association between ultrasound findings and clinical and functional outcome measures including pain VAS and FIHOA. This suggests that ultrasound findings do not sufficiently reflect the clinical features and functional status of hand OA. This inconsistency can be explained by the following reasons. First, subclinical or low-grade inflammation and structural bone changes in affected joints detected by ultrasound precede development of pain or functional impairment. Second, OA is due to a consequence of multifactorial etiology, so it is fundamentally difficult to fully understand the clinical symptoms by ultrasound findings. Thus, in the evaluation of hand OA, ultrasound examination should play a complementary role to clinical findings and functional examination.

It is well known that grip and pinch strengths were significantly lower in patients with hand $\mathrm{OA}$ and were significantly associated with severity of hand OA [26,27]. Jones et al. [28] proposed that hand power was dependent on pain in hand OA. However, the relationship of grip and pinch strength with radiographic severity was not found in a study of middle-aged female dentists and teachers [26]. Our study also could not find any ultrasound findings that had a relationship with grip and pinch strength, which is consistent with the results of an earlier study [10]. In contrast, Naguib et al. [14] demonstrated negative correlation of grip and pinch strength with cartilage thinning and osteophytes on ultrasound. Whether ultrasound findings are associated with grip and pinch strength remains debatable; further research on this relationship should be conducted with a large study population. Considering the relationship between ultrasound path- ologies and hand functional status, ultrasound-detected synovitis, effusion, osteophytes, or joint space narrowing previously was found to be associated with one of the functional indexes, The AUStralian CANadian Osteoarthritis Hand Index (AUSCAN) scores [11,28], which was inconsistent with the results in our study. We found that statistical significance for association of osteophytes in ultrasound with grip strength was lost after adjustment for confounding variables. Similarly, functional measures such as AUSCAN and FIHOA did not have significant association with ultrasound findings $[10,12,13]$. It is thought that the inconsistency in results of these studies is related to the characteristics of the study groups, such as sex, age, disease severity, distribution of affected joints, and heterogeneity of diagnostic criteria.

This study has several limitations. First, it seems that the small number of patients is a major limitation. Our study was similar or slightly larger than the sample size in most of the similar studies that analyzed relevance [11-14]. Post hoc power analysis showed that our sample size was appropriate for association between ultrasound findings and hand OA-related symptoms, function, and radiographic changes. Second, our study did not consider confounding factors that affect hand OA, such as occupation, economic status, and lifestyle. Bedson and Croft [29] postulated that the discrepancy between clinical and radiographic changes in knee OA was mediated by pain definition and the nature of the study population. Similarly, psychosomatic behavior, occupation, and living style, which are able to affect pain or radiographic progression, should be considered.

\section{CONCLUSION}

Earlier studies of the association between ultrasound-detected abnormalities and clinical symptoms, functional status, or radiographic features in hand $\mathrm{OA}$ have some debatable conclusions [10-14]. Our study confirmed a significant correlation between ultrasound-detected osteophytes and K-L grade-based radiographic scores, consistent with results in other studies $[10,14]$. Although the statistical correlation was weak, patients with severe radiographic changes on conventional radiography suggest that inflammatory changes on ultrasound are likely to be increased. We demonstrated that the clinical symptoms and functional disability were not related to the ultrasound findings. However, additional studies are needed to verify the relevance between these measures. 


\section{CONFLICT OF INTEREST}

No potential conflict of interest relevant to this article was reported.

\section{AUTHOR CONTRIBUTIONS}

Design of this study: K.S.K. Interpretation and statistical analysis of data: J.Y.C., U.H.J., J.W.K. and K.S.K. Collection of data: U.H.J., J.W.K., K.S.K. Drafting the manuscript: K.S.K. Approval of final manuscript: J.Y.C., U.H.J., J.W.K. and K.S.K.

\section{REFERENCES}

1. Zhang W, Doherty M, Leeb BF, Alekseeva L, Arden NK, Bijlsma JW, et al. EULAR evidence-based recommendations for the diagnosis of hand osteoarthritis: report of a task force of ESCISIT. Ann Rheum Dis 2009;68:8-17.

2. Marshall M, Watt FE, Vincent TL, Dziedzic K. Hand osteoarthritis: clinical phenotypes, molecular mechanisms and disease management. Nat Rev Rheumatol 2018;14:641-56.

3. Altman R, Alarcón G, Appelrouth D, Bloch D, Borenstein D, Brandt K, et al. The American College of Rheumatology criteria for the classification and reporting of osteoarthritis of the hand. Arthritis Rheum 1990;33:1601-10.

4. Dahaghin S, Bierma-Zeinstra SM, Ginai AZ, Pols HA, Hazes JM, Koes BW. Prevalence and pattern of radiographic hand osteoarthritis and association with pain and disability (the Rotterdam study). Ann Rheum Dis 2005;64:682-7.

5. Haugen IK, Englund M, Aliabadi P, Niu J, Clancy M, Kvien TK, et al. Prevalence, incidence and progression of hand osteoarthritis in the general population: the Framingham Osteoarthritis Study. Ann Rheum Dis 2011;70:1581-6.

6. Bellamy N, Kirwan J, Boers M, Brooks P, Strand V, Tugwell $\mathrm{P}$, et al. Recommendations for a core set of outcome measures for future phase III clinical trials in knee, hip, and hand osteoarthritis. Consensus development at OMERACT III. J Rheumatol 1997;24:799-802.

7. Maheu E, Altman RD, Bloch DA, Doherty M, Hochberg M, Mannoni A, et al. Design and conduct of clinical trials in patients with osteoarthritis of the hand: recommendations from a task force of the Osteoarthritis Research Society International. Osteoarthritis Cartilage 2006;14:303-22.

8. Haugen IK, Bøyesen P. Imaging modalities in hand osteoarthritis--and perspectives of conventional radiography, magnetic resonance imaging, and ultrasonography. Arthritis Res Ther 2011;13:248.

9. McNally EG. Ultrasound of the small joints of the hands and feet: current status. Skeletal Radiol 2008;37:99-113.

10. Oo WM, Deveza LA, Duong V, Fu K, Linklater JM, Riordan EA, et al. Musculoskeletal ultrasound in symptomatic thumb-base osteoarthritis: clinical, functional, radiological and muscle strength associations. BMC Musculoskelet Disord 2019;20:220.

11. Kortekaas MC, Kwok WY, Reijnierse M, Watt I, Huizinga
TW, Kloppenburg M. Pain in hand osteoarthritis is associated with inflammation: the value of ultrasound. Ann Rheum Dis 2010;69:1367-9.

12. Keen HI, Wakefield RJ, Grainger AJ, Hensor EM, Emery P, Conaghan PG. An ultrasonographic study of osteoarthritis of the hand: synovitis and its relationship to structural pathology and symptoms. Arthritis Rheum 2008;59:1756-63.

13. Koutroumpas AC, Alexiou IS, Vlychou M, Sakkas LI. Comparison between clinical and ultrasonographic assessment in patients with erosive osteoarthritis of the hands. Clin Rheumatol 2010;29:511-6.

14. Naguib A, Mohasseb D, Sultan H, Hamimi A, Fawzy M. Hand osteoarthritis: clinical and imaging study. Alex J Med 2011;47:237-42.

15. Dreiser RL, Maheu E, Guillou GB, Caspard H, Grouin JM. Validation of an algofunctional index for osteoarthritis of the hand. Rev Rhum Engl Ed 1995;62(6 Suppl 1): 43S-53S.

16. Ahn GY, Cho SK, Cha SJ, Nam E, Lee JE, Dreiser RL, et al. Cross-cultural adaptation and validation of the Korean Version of the Functional Index for Hand Osteoarthritis (FIHOA). Int J Rheum Dis 2018;21:2095-103.

17. Huskisson EC. Measurement of pain. Lancet 1974;2:1127-31.

18. Kellgren JH, Lawrence JS. Radiological assessment of osteo-arthrosis. Ann Rheum Dis 1957;16:494-502.

19. Bijsterbosch J, Visser W, Kroon HM, Stamm T, Meulenbelt I, Huizinga TW, et al. Thumb base involvement in symptomatic hand osteoarthritis is associated with more pain and functional disability. Ann Rheum Dis 2010;69:585-7.

20. Kim SK, Jung UH, Choe JY. Clinical Usefulness of uric acid as a biomarker for knee osteoarthritis: a comparative analysis with plain radiography and musculoskeletal ultrasound. J Rheum Dis 2020;27:51-60.

21. Roux CH, Foltz V, Maheu E, Baron G, Gandjbakhch F, Lukas $\mathrm{C}$, et al. MRI and serum biomarkers correlate with radiographic features in painful hand osteoarthritis. Clin Exp Rheumatol 2016;34:991-8.

22. van der Kraan PM, van den Berg WB. Osteophytes: relevance and biology. Osteoarthritis Cartilage 2007; 15:237-44.

23. Visser AW, Bøyesen P, Haugen IK, Schoones JW, van der Heijde DM, Rosendaal FR, et al. Radiographic scoring methods in hand osteoarthritis--a systematic literature search and descriptive review. Osteoarthritis Cartilage 2014;22: 1710-23.

24. Lawrence JS, Bremner JM, Bier F. Osteo-arthrosis. Prevalence in the population and relationship between symptoms and x-ray changes. Ann Rheum Dis 1966;25:1-24.

25. Hart D, Spector T, Egger P, Coggon D, Cooper C. Defining osteoarthritis of the hand for epidemiological studies: the Chingford Study. Ann Rheum Dis 1994;53:220-3.

26. Ding H, Solovieva S, Vehmas T, Takala EP, Leino-Arjas P. Hand osteoarthritis and pinch grip strength among middle-aged female dentists and teachers. Scand J Rheumatol 2010;39:84-7.

27. Bagis S, Sahin G, Yapici Y, Cimen OB, Erdogan C. The effect of hand osteoarthritis on grip and pinch strength and hand function in postmenopausal women. Clin Rheumatol 2003; 22:420-4.

28. Jones G, Cooley HM, Bellamy N. A cross-sectional study of the association between Heberden's nodes, radiographic os- 
teoarthritis of the hands, grip strength, disability and pain. Osteoarthritis Cartilage 2001;9:606-11.

29. Bedson J, Croft PR. The discordance between clinical and ra- diographic knee osteoarthritis: a systematic search and summary of the literature. BMC Musculoskelet Disord 2008;9:116. 